\title{
Properties and Riemann-Liouville
}

\section{fractional Hermite-Hadamard inequalities for the generalized $(\alpha, m)$-preinvex functions}

\author{
TingSong Du ${ }^{*}$, JiaGen Liao' , LianZi Chen ${ }^{1}$ and Muhammad Uzair Awan²
}

\section{"Correspondence:}

tingsongdu@ctgu.edu.cn

'Department of Mathematics, College of Science, China Three

Gorges University, Yichang, 443002, China

Full list of author information is available at the end of the article

\begin{abstract}
The authors first introduce the concepts of generalized $(\alpha, m)$-preinvex function, generalized quasi $m$-preinvex function and explicitly $(\alpha, m)$-preinvex function, and then provide some interesting properties for the newly introduced functions. The more important point is that we give a necessary and sufficient condition respecting the relationship between the generalized $(\alpha, m)$-preinvex function and the generalized quasi $m$-preinvex function. Second, a new Riemann-Liouville fractional integral identity involving twice differentiable function on $m$-invex is found. By using this identity, we establish the right-sided new Hermite-Hadamard-type inequalities via Riemann-Liouville fractional integrals for generalized $(\alpha, m)$-preinvex mappings. These inequalities can be viewed as generalization of several previously known results.
\end{abstract}

MSC: 26A33; 26A51; 26D07; 26D20; 41A55

Keywords: Hermite-Hadamard's inequality; $(\alpha, m)$-preinvex functions; Riemann-Liouville fractional integrals

\section{Introduction}

The following notation is used throughout this paper. We use $I$ to denote an interval on the real line $\mathbb{R}=(-\infty, \infty)$. For any subset $K \subseteq \mathbb{R}^{n}, K^{\circ}$ is used to denote the interior of $K$ and $\mathbb{R}^{n}$ is used to denote a generic $n$-dimensional vector space. The set of integrable functions on the interval $[a, b]$ is denoted by $L^{1}[a, b]$. The non-negative real numbers and the positive real numbers are denoted by $\mathbb{R}_{0}=[0, \infty)$ and $\mathbb{R}_{+}=(0, \infty)$, respectively.

Let $f: I \subseteq \mathbb{R} \rightarrow \mathbb{R}$ be a convex mapping defined on the interval $I$ of real numbers and $a, b \in I$ with $a<b$. The inequality

$$
f\left(\frac{a+b}{2}\right) \leq \frac{1}{b-a} \int_{a}^{b} f(x) \mathrm{d} x \leq \frac{f(a)+f(b)}{2}
$$

referred to as Hermite-Hadamard inequality, is one of the most famous results for convex mappings. A number of papers have been written on this inequality providing new proofs, noteworthy extensions, generalizations, refinements and new inequalities connected with the Hermite-Hadamard inequality. The reader may refer to [1-9] and the references therein.

We need, now, some necessary definitions and preliminary results as follows.

(c) The Author(s) 2016. This article is distributed under the terms of the Creative Commons Attribution 4.0 International License (http://creativecommons.org/licenses/by/4.0/), which permits unrestricted use, distribution, and reproduction in any medium, provided you give appropriate credit to the original author(s) and the source, provide a link to the Creative Commons license, and indicate if changes were made. 
Definition 1.1 ([10,11]) A set $S \subseteq \mathbb{R}^{n}$ is said to be invex set with respect to the mapping $\eta: S \times S \rightarrow \mathbb{R}^{n}$ if $x+t \eta(y, x) \in S$ for every $x, y \in S$ and $t \in[0,1]$. The invex set $S$ is also called an $\eta$-connected set.

Notice that every convex set is invex with respect to the mapping $\eta(y, x)=y-x$, but the converse is not necessarily true. See [10], for example.

Definition 1.2 ([12]) A set $K \subseteq \mathbb{R}^{n}$ is said to be $m$-invex with respect to the mapping $\eta: K \times K \times(0,1] \rightarrow \mathbb{R}^{n}$ for some fixed $m \in(0,1]$, if $m x+\lambda \eta(y, x, m) \in K$ holds for each $x, y \in K$ and any $\lambda \in[0,1]$.

The Definition 1.2 essentially says that there is a path for some fixed $m \in(0,1]$, starting from $m x$, which is contained in $K$. We do not require that $y$ should be one of the end points of the path. However, if we demand that $y$ should be an end point of the path for every pair $x, y$, then $\eta(y, x, m)=y-m x$ with $m=1$, reducing to convexity.

It is noticed that every convex set is $m$-invex with respect to the mapping $\eta(y, x, m)=$ $y-m x$ with $m=1$, but the converse is not necessarily true. See [12], for example.

Definition 1.3 ([13]) The function $f:[0, b] \rightarrow \mathbb{R}, b>0$, is said to be $(\alpha, m)$-convex where $(\alpha, m) \in(0,1] \times(0,1]$, if we have

$$
f(t x+m(1-t) y) \leq t^{\alpha} f(x)+m\left(1-t^{\alpha}\right) f(y)
$$

for all $x, y \in[0, b]$ and $t \in[0,1]$.

Definition 1.4 ([11]) The function $f$ defined on the invex set $K \subseteq \mathbb{R}^{n}$ is said to be preinvex with respect to $\eta$ if for every $x, y \in K$ and $t \in[0,1]$, we have

$$
f(x+t \eta(y, x)) \leq(1-t) f(x)+t f(y) .
$$

The function $f$ is said to be preconcave if and only if $-f$ is preinvex.

The concept of preinvexity is more general than convexity since every convex function is preinvex with respect to the mapping $\eta(y, x)=y-x$. Further, there exist preinvex mappings which are not convex.

Theorem 1.1 ([14]) Let $f: K=[a, a+\eta(b, a)] \rightarrow(0, \infty)$ be a preinvex function on the interval of the real numbers $K^{\circ}$ and $a, b \in K^{\circ}$ with $\eta(b, a)>0$. Then the following inequality holds:

$$
f\left(\frac{2 a+\eta(b, a)}{2}\right) \leq \frac{1}{\eta(b, a)} \int_{a}^{a+\eta(b, a)} f(x) \mathrm{d} x \leq \frac{f(a)+f(b)}{2} .
$$

The inequality (1.4) is usually termed the Hermite-Hadamard-Noor-type inequality for preinvex mappings. This result is analogous to the original Hermite-Hadamard inequalities. If $\eta(b, a)=b-a$, then the inequality (1.4) reduces to the remarkable HermiteHadamard's inequality (1.1). 
For recent results on some new generalizations, refinements of integral inequalities involved with the preinvex functions, one can see [12, 15-18] and the references therein.

In [19], Latif and Shoaib raised the so-called $(\alpha, m)$-preinvex function below.

Definition 1.5 ([19]) The function $f$ on the invex set $K \subseteq\left[0, b^{*}\right], b^{*}>0$, is said to be $(\alpha, m)$-preinvex with respect to $\eta$ if

$$
f(x+t \eta(y, x)) \leq\left(1-t^{\alpha}\right) f(x)+m t^{\alpha} f\left(\frac{y}{m}\right) .
$$

holds for all $x, y \in K, t \in[0,1]$ and $(\alpha, m) \in(0,1] \times(0,1]$. The function $f$ is said to be $(\alpha, m)$ preincave if and only if $-f$ is $(\alpha, m)$-preinvex.

We also need the following fractional calculus background.

Definition 1.6 ([20]) Let $f \in L^{1}[a, b]$. The left-sided and right-sided Riemann-Liouville fractional integrals of order $\alpha>0$ with $a \geq 0$ are defined by

$$
J_{a^{+}}^{\alpha} f(x)=\frac{1}{\Gamma(\alpha)} \int_{a}^{x}(x-t)^{\alpha-1} f(t) \mathrm{d} t, \quad a<x,
$$

and

$$
J_{b}^{\alpha} f(x)=\frac{1}{\Gamma(\alpha)} \int_{x}^{b}(t-x)^{\alpha-1} f(t) \mathrm{d} t, \quad x<b
$$

respectively, where $\Gamma(\cdot)$ is Gamma function and its definition is $\Gamma(\alpha)=\int_{0}^{\infty} e^{-u} u^{\alpha-1} \mathrm{~d} u$. It is to be noted that $J_{a^{+}}^{0} f(x)=J_{b-}^{0} f(x)=f(x)$.

In the case $\alpha=1$, the Riemann-Liouville fractional integral becomes the classical integral.

In [21], Sarikaya et al. established the following interesting inequalities of HermiteHadamard-type involving Riemann-Liouville fractional integrals.

Theorem 1.2 Let $f:[a, b] \rightarrow \mathbb{R}$ be a positive function with $0 \leq a<b$ and $f \in L^{1}[a, b]$. If $f$ is a convex function on $[a, b]$, then the following inequalities for fractional integrals hold:

$$
f\left(\frac{a+b}{2}\right) \leq \frac{\Gamma(\alpha+1)}{2(b-a)^{\alpha}}\left[J_{a^{+}}^{\alpha} f(b)+J_{b^{-}}^{\alpha} f(a)\right] \leq \frac{f(a)+f(b)}{2}
$$

with $\alpha>0$.

Observe that, for $\alpha=1$, the inequalities (1.6) becomes the original Hermite-Hadamard inequality (1.1).

For some recent results associated with the fractional integral inequalities, one can consult [22-32].

In a very recently published paper [33] by Hussain and Qaisar, they found some HermiteHadamard integral inequalities for mapping whose absolute values of derivatives are $(\alpha, m)$-preinvex, and in the article [34] by Qaisar et al., they also obtained Riemann- 
Liouville fractional Hadamard-type integral inequalities for mappings whose absolute value of first derivatives are preinvex.

Motivated by this idea and based on our previous work $[2,12,17,35,36]$, in the present paper, the next section we are going to introduce new concepts, to be referred as the generalized $(\alpha, m)$-preinvex function, the generalized quasi $m$-preinvex function and the explicitly $(\alpha, m)$-preinvex function, respectively, and then we derive some interesting properties for the newly introduced functions. In this section, the more important point is that we give a necessary and sufficient condition with respect to the relationship between the generalized $(\alpha, m)$-preinvex function and the generalized quasi $m$-preinvex function. In Section 3, we will discover a Riemann-Liouville fractional integral identity involving twice differentiable preinvex functions. By using this identity, we explore the right-sided new Hermite-Hadamard-type inequalities for mappings whose absolute value of second derivatives are generalized $(\alpha, m)$-preinvex via Riemann-Liouville fractional integrals. These inequalities can be viewed as generalization of the results of $[37,38]$.

\section{New definitions and properties}

As one can see, the definitions of the preinvex, $(\alpha, m)$-convex, and $(\alpha, m)$-preinvex mappings have similar configurations. This observation leads us to generalize these varieties of convexity.

We next give new definitions, to be referred to as the generalized $(\alpha, m)$-preinvex function, the generalized quasi $m$-preinvex function and the explicitly $(\alpha, m)$-preinvex function, respectively.

Definition 2.1 Let $K \subseteq \mathbb{R}^{n}$ be an open $m$-invex set with respect to $\eta: K \times K \times(0,1] \rightarrow \mathbb{R}^{n}$.

(i) For $f: K \rightarrow \mathbb{R}$ and some fixed $\alpha, m \in(0,1]$, if

$$
f(m x+\lambda \eta(y, x, m)) \leq m\left(1-\lambda^{\alpha}\right) f(x)+\lambda^{\alpha} f(y)
$$

is valid for all $x, y \in K, \lambda \in[0,1]$, then we say that $f(x)$ is a generalized

$(\alpha, m)$-preinvex function with respect to $\eta$.

(ii) For $f: K \rightarrow \mathbb{R}$ and some fixed $m \in(0,1]$, if

$$
f(m x+\lambda \eta(y, x, m)) \leq \max \{f(x), f(y)\}
$$

is valid for all $x, y \in K, \lambda \in[0,1]$, then we say that $f(x)$ is a generalized quasi $m$-preinvex function with respect to $\eta$.

The function $f(x)$ is said to be strictly generalized $(\alpha, m)$-preinvex function on $K$ with respect to $\eta$, if a strict inequality holds on (2.1) for any $x, y \in K$ and $x \neq y$.

Remark 2.1 In Definition 2.1, it is worthwhile to note that generalized $(\alpha, m)$-preinvex function is an $(\alpha, m)$-convex function on $K$ with respect to $\eta(y, x, m)=y-m x$.

Definition 2.2 Let $K \subseteq \mathbb{R}^{n}$ be an open $m$-invex set with respect to $\eta: K \times K \times(0,1] \rightarrow \mathbb{R}^{n}$. For $f: K \rightarrow \mathbb{R}$ and some fixed $\alpha, m \in(0,1]$, if $\forall \lambda \in(0,1), \forall x, y \in K$ and $f(x) \neq f(y)$, we have

$$
f(m x+\lambda \eta(y, x, m))<m\left(1-\lambda^{\alpha}\right) f(x)+\lambda^{\alpha} f(y),
$$

then we say that $f(x)$ is an explicitly $(\alpha, m)$-preinvex function with respect to $\eta$. 
Example 2.1 Let $f(x)=\sin x, \alpha=1$, and let

$$
\eta(y, x, m)= \begin{cases}\frac{\sin y-m \sin x}{m \cos x}, & y \geq x \\ 0, & y<x .\end{cases}
$$

Then $f(x)$ is a generalized $\left(1, \frac{1}{2}\right)$-preinvex function with respect to $\eta: \mathbb{R} \times \mathbb{R} \times(0,1] \rightarrow \mathbb{R}$. However, it is obvious that $f(x)=\sin x$ is not a convex function on $\mathbb{R}$. By letting $x>y=$ $\frac{\pi}{2}, \lambda=\frac{1}{2}$, we have

$$
f(m x+\lambda \eta(y, x, m))=f\left(\frac{1}{2} x+\frac{1}{2} \eta\left(\frac{\pi}{2}, x, m\right)\right)=\sin \left(\frac{1}{2} x\right)
$$

and

$$
m\left(1-\lambda^{\alpha}\right) f(x)+\lambda^{\alpha} f(y)=\frac{1}{4} \sin x+\frac{1}{2} .
$$

Thus, there must exist an $x_{0}>y=\frac{\pi}{2}$ such that $f\left(x_{0}\right) \neq f(y)=f\left(\frac{\pi}{2}\right)=1$ and

$$
\sin \left(\frac{1}{2} x_{0}\right)=\frac{1}{4} \sin x_{0}+\frac{1}{2}
$$

Hence, $f$ is not also an explicitly $(\alpha, m)$-preinvex function on $\mathbb{R}$ with respect to $\eta$ for $\alpha=1$ and $m=\frac{1}{2}$.

The so-called 'generalized $(\alpha, m)$-logarithmically preinvexity', may be introduced as follows.

Definition 2.3 Let $K \subseteq \mathbb{R}^{n}$, be an open $m$-invex set with respect to $\eta: K \times K \times(0,1] \rightarrow \mathbb{R}^{n}$. For $f: K \rightarrow \mathbb{R}_{+}$and some fixed $\alpha, m \in(0,1]$, if $\forall \lambda \in(0,1), \forall x, y \in K$, we have

$$
f(m x+\lambda \eta(y, x, m)) \leq[f(x)]^{m\left(1-\lambda^{\alpha}\right)}[f(y)]^{\lambda^{\alpha}},
$$

then we say that $f(x)$ is a generalized $(\alpha, m)$-logarithmically preinvex function with respect to $\eta$.

Based on the above Definition 2.1 and Definition 2.2, we investigate, now, some interesting properties of the generalized $(\alpha, m)$-preinvex function, generalized quasi $m$-preinvex function and explicitly $(\alpha, m)$-preinvex function. The first observation is given as follows.

Proposition 2.1 Iff $: K \subseteq \mathbb{R}^{n} \rightarrow \mathbb{R}_{0}$ is a generalized ( $\left.\alpha, m\right)$-preinvex function on m-invex set $K$ with respect to $\eta$, then $f$ is also a generalized quasi m-preinvex function on $m$-invex set $K$ with respect to $\eta$.

Proof Since $f$ is a non-negative generalized $(\alpha, m)$-preinvex function, we assume that $f(x) \leq f(y), \forall x, y \in K$, for every $\lambda \in[0,1]$, we have

$$
f(m x+\lambda \eta(y, x, m)) \leq m\left(1-\lambda^{\alpha}\right) f(x)+\lambda^{\alpha} f(y) \leq\left[m\left(1-\lambda^{\alpha}\right)+\lambda^{\alpha}\right] f(y) \leq f(y) .
$$


In the same way, let $f(y) \leq f(x), \forall x, y \in K$, we can also get

$$
f(m x+\lambda \eta(y, x, m)) \leq f(x)
$$

Consequently,

$$
f(m x+\lambda \eta(y, x, m)) \leq \max \{f(x), f(y)\} .
$$

That is, $f$ is a generalized quasi $m$-preinvex function on $m$-invex set $K$ with respect to $\eta$, the required result.

The proofs of Propositions 2.2 and 2.3 are all easy to verify.

Proposition 2.2 If $f_{i}: K \subseteq \mathbb{R}^{n} \rightarrow \mathbb{R}(i=1,2, \ldots, n)$ are generalized $(\alpha, m)$-preinvex (explicitly $(\alpha, m)$-preinvex) functions on $m$-invex set $K$ with respect to the same $\eta: K \times K \times(0,1] \rightarrow$ $\mathbb{R}$ for same fixed $\alpha, m \in(0,1]$, then the function

$$
f=\sum_{i=1}^{n} a_{i} f_{i}, a_{i} \geq 0 \quad(i=1,2, \ldots, n)
$$

is also a generalized $(\alpha, m)$-preinvex (explicitly $(\alpha, m)$-preinvex) functions on $m$-invex set $K$ with respect to the same $\eta$ for fixed $\alpha, m \in(0,1]$.

Proposition 2.3 If $f_{i}: K \subseteq \mathbb{R}^{n} \rightarrow \mathbb{R}(i=1,2, \ldots, n)$ are generalized $(\alpha, m)$-preinvex (explicitly $(\alpha, m)$-preinvex) functions on $m$-invex set $K$ with respect to the same $\eta: K \times K \times(0,1] \rightarrow$ $\mathbb{R}$ for same fixed $\alpha, m \in(0,1]$, then the function

$$
f=\max \left\{f_{i}, i=1,2, \ldots, n\right\}
$$

is also a generalized $(\alpha, m)$-preinvex (an explicitly $(\alpha, m)$-preinvex) function on m-invex set $K$ with respect to the same $\eta$ for fixed $\alpha, m \in(0,1]$.

In Proposition 2.4 we prove that the combination of a generalized $(\alpha, m)$-preinvex function with a sublinear and nondecreasing function is a generalized $(\alpha, m)$-preinvex function.

Proposition 2.4 Let $K$ be a nonempty m-invex set in $\mathbb{R}^{n}$ with respect to $\eta: K \times K \times(0,1] \rightarrow$ $\mathbb{R}^{n}, f: K \rightarrow \mathbb{R}$ be a generalized $(\alpha, m)$-preinvex function with respect to $\eta$ for some fixed $\alpha, m \in(0,1]$, and let $g: W \rightarrow \mathbb{R}(W \subseteq \mathbb{R})$ be a sublinear and nondecreasing function, where $\operatorname{rang}(f) \subseteq W$. Then the composite function $g(f)$ is a generalized $(\alpha, m)$-preinvex function with respect to $\eta$ on $K$ for fixed $\alpha, m \in(0,1]$.

Proof Since $f$ is a generalized $(\alpha, m)$-preinvex function, for all $x, y \in K$, we have

$$
f(m x+\lambda \eta(y, x, m)) \leq m\left(1-\lambda^{\alpha}\right) f(x)+\lambda^{\alpha} f(y)
$$

holds for any $\lambda \in[0,1]$. Notice that $g$ is a sublinear and nondecreasing function, it yields

$$
g(f(m x+\lambda \eta(y, x, m))) \leq g\left(m\left(1-\lambda^{\alpha}\right) f(x)+\lambda^{\alpha} f(y)\right) \leq m\left(1-\lambda^{\alpha}\right) g(f(x))+\lambda^{\alpha} g(f(y)),
$$


from which it follows that $g(f)$ is a generalized $(\alpha, m)$-preinvex function with respect to $\eta$ on $K$ for some fixed $\alpha, m \in(0,1]$.

Proposition 2.5 Let $K$ be a nonempty m-invex set in $\mathbb{R}^{n}$ with respect to $\eta: K \times K \times(0,1] \rightarrow$ $\mathbb{R}^{n}$, and $f, g: K \rightarrow \mathbb{R}$ be generalized $(\alpha, m)$-preinvex functions with respect to the same $\eta$ for some fixed $\alpha, m \in(0,1]$. Then their product fg is also a generalized $(\alpha, m)$-preinvex function provided that $f$ and $g$ are similarly ordered functions with $f g \geq 0$.

Proof Since $f$ and $g$ are two similarly ordered generalized $(\alpha, m)$-preinvex functions, we have

$$
\begin{aligned}
f( & m x+\lambda \eta(y, x, m)) g(m x+\lambda \eta(y, x, m)) \\
\quad \leq & {\left[m\left(1-\lambda^{\alpha}\right) f(x)+\lambda^{\alpha} f(y)\right]\left[m\left(1-\lambda^{\alpha}\right) g(x)+\lambda^{\alpha} g(y)\right] } \\
\quad= & {\left[m\left(1-\lambda^{\alpha}\right)\right]^{2} f(x) g(x)+\left(\lambda^{\alpha}\right)^{2} f(y) g(y)+m\left(1-\lambda^{\alpha}\right) \lambda^{\alpha}[f(x) g(y)+f(y) g(x)] } \\
& \leq\left[m\left(1-\lambda^{\alpha}\right)\right]^{2} f(x) g(x)+\left(\lambda^{\alpha}\right)^{2} f(y) g(y)+m\left(1-\lambda^{\alpha}\right) \lambda^{\alpha}[f(x) g(x)+f(y) g(y)] \\
& =m\left(1-\lambda^{\alpha}\right)\left[m\left(1-\lambda^{\alpha}\right)+\lambda^{\alpha}\right] f(x) g(x)+\lambda^{\alpha}\left[m\left(1-\lambda^{\alpha}\right)+\lambda^{\alpha}\right] f(y) g(y) \\
& \leq m\left(1-\lambda^{\alpha}\right) f(x) g(x)+\lambda^{\alpha} f(y) g(y),
\end{aligned}
$$

where we used the required condition $f g \geq 0$. This shows that the product of two generalized $(\alpha, m)$-preinvex functions is also a generalized $(\alpha, m)$-preinvex function.

Proposition 2.6 If $g_{i}: \mathbb{R}^{n} \rightarrow \mathbb{R}(i=1,2, \ldots, n)$ are generalized $(\alpha, m)$-preinvex functions with respect to the same $\eta$ for same fixed $\alpha, m \in(0,1]$, then the set $M=\left\{x \in \mathbb{R}^{n}: g_{i}(x) \leq\right.$ $0, i=1,2, \ldots, n\}$ is an m-invex set.

Proof Since $g_{i}(x)(i=1,2, \ldots, n)$ are generalized $(\alpha, m)$-preinvex functions, for all $x, y \in \mathbb{R}^{n}$, we have

$$
g_{i}(m x+\lambda \eta(y, x, m)) \leq m\left(1-\lambda^{\alpha}\right) g_{i}(y)+\lambda^{\alpha} g_{i}(x), \quad i=1,2, \ldots, n,
$$

holds for any $\lambda \in[0,1]$. When $x, y \in M$, we know $g_{i}(x) \leq 0$ and $g_{i}(y) \leq 0$. From the above inequality, it yields

$$
g_{i}(m x+\lambda \eta(y, x, m)) \leq 0, \quad i=1,2, \ldots, n .
$$

That is, $m x+\lambda \eta(y, x, m) \in M$. Hence, $M$ is an $m$-invex set.

Proposition 2.7 Let $f: \mathbb{R}_{0} \rightarrow \mathbb{R}_{0}$ is a generalized $(\alpha, m)$-preinvex function with respect to $\eta: \mathbb{R}_{0} \times \mathbb{R}_{0} \times(0,1] \rightarrow \mathbb{R}_{0}$ for some fixed $\alpha, m \in(0,1]$. Assume that $f$ is monotone decreasing, $\eta$ is monotone increasing regarding $m$ for fixed $x, y \in \mathbb{R}_{0}$, and $m_{1} \leq m_{2}\left(m_{1}, m_{2} \in(0,1]\right)$. Iff is a generalized $\left(\alpha, m_{1}\right)$-preinvex function on $\mathbb{R}_{0}$ with respect to $\eta$, then $f$ is also a generalized $\left(\alpha, m_{2}\right)$-preinvex function on $\mathbb{R}_{0}$ with respect to $\eta$.

Proof Since $f$ is a generalized $\left(\alpha, m_{1}\right)$-preinvex function, for all $x, y \in \mathbb{R}_{0}$, we have

$$
f\left(m_{1} x+\lambda \eta\left(y, x, m_{1}\right)\right) \leq m_{1}\left(1-\lambda^{\alpha}\right) f(x)+\lambda^{\alpha} f(y) .
$$


Combining the monotone decreasing of the function $f$ with the monotone increasing of the mapping $\eta$ regarding $m$ for fixed $x, y \in \mathbb{R}_{0}$, and $m_{1} \leq m_{2}$, it follows that

$$
f\left(m_{2} x+\lambda \eta\left(y, x, m_{2}\right)\right) \leq f\left(m_{1} x+\lambda \eta\left(y, x, m_{1}\right)\right)
$$

and

$$
m_{1}\left(1-\lambda^{\alpha}\right) f(x)+\lambda^{\alpha} f(y) \leq m_{2}\left(1-\lambda^{\alpha}\right) f(x)+\lambda^{\alpha} f(y)
$$

Following the above two inequalities, we have

$$
f\left(m_{2} x+\lambda \eta\left(y, x, m_{2}\right)\right) \leq m_{2}\left(1-\lambda^{\alpha}\right) f(x)+\lambda^{\alpha} f(y) .
$$

Hence, $f$ is also a generalized $\left(\alpha, m_{2}\right)$-preinvex function on $\mathbb{R}_{0}$ with respect to $\eta$ for fixed $\alpha \in(0,1]$, which ends the proof.

Proposition 2.8 Let $K$ be a nonempty m-invex set in $\mathbb{R}^{n}$ with respect to $\eta: K \times K \times(0,1] \rightarrow$ $\mathbb{R}^{n}$, and $f_{i}: K \rightarrow \mathbb{R}(i \in I=\{1,2, \ldots, n\})$ be a family of real-valued fucntions which are explicitly $(\alpha, m)$-preinvex functions with respect to the same $\eta$ for same fixed $\alpha, m \in(0,1]$ and bounded from above on $K$. Then the function $f(x)=\sup \left\{f_{i}(x), i \in I\right\}$ is also an explicitly $(\alpha, m)$-preinvex function on $K$ with respect to the same $\eta$ for fixed $\alpha, m \in(0,1]$.

Proof Since each $f_{i}(x)(i \in I)$ is an explicitly $(\alpha, m)$-preinvex function with respect to the same $\eta$ for same fixed $\alpha, m \in(0,1]$, we have for each $i \in I$

$$
f_{i}(m x+\lambda \eta(y, x, m))<m\left(1-\lambda^{\alpha}\right) f_{i}(x)+\lambda^{\alpha} f_{i}(y), \quad \forall x, y \in K, \lambda \in(0,1) .
$$

Therefore, for each $i \in I$,

$$
f_{i}(m x+\lambda \eta(y, x, m))<m\left(1-\lambda^{\alpha}\right) \sup _{i \in I} f_{i}(x)+\lambda^{\alpha} \sup _{i \in I} f_{i}(y), \quad \forall x, y \in K, \lambda \in(0,1) .
$$

Taking the sup of the left-hand side of the above inequality, we obtain

$$
\sup _{i \in I} f_{i}(m x+\lambda \eta(y, x, m))<m\left(1-\lambda^{\alpha}\right) \sup _{i \in I} f_{i}(x)+\lambda^{\alpha} \sup _{i \in I} f_{i}(y), \quad \forall x, y \in K, \lambda \in(0,1) .
$$

That is, $f(x)=\sup \left\{f_{i}(x), i \in I\right\}$ is also an explicitly $(\alpha, m)$-preinvex function on $K$ with respect to the same $\eta$ for fixed $\alpha, m \in(0,1]$.

Proposition 2.9 below reveals that a local minimum of an explicitly $(\alpha, m)$-preinvex function on an $m$-invex set is a global one under some conditions.

Proposition 2.9 Let $K$ be a nonempty m-invex set in $\mathbb{R}^{n}$ with respect to $\eta: K \times K \times(0,1] \rightarrow$ $\mathbb{R}^{n}$, and $f: K \rightarrow \mathbb{R}_{0}$ be an explicitly $(\alpha, m)$-preinvex function with respect to $\eta$ for some fixed $\alpha, m \in(0,1]$. If $\bar{x} \in K$ is a local minimum to the problem of minimizing $f(x)$ subject to $x \in K$, then $\bar{x}$ is a global one. 
Proof Suppose that $\bar{x} \in K$ is a local minimum to the problem of minimizing $f(x)$ subject to $x \in K$. Then there is an $\varepsilon$-neighborhood $N_{\varepsilon}(\bar{x})$ around $\bar{x}$ such that

$$
f(\bar{x}) \leq f(x), \quad \forall x \in K \cap N_{\varepsilon}(\bar{x}) .
$$

If $\bar{x}$ is not global minimum of $f(x)$ on $K$, then there exists an $x^{*} \in K$ such that

$$
f\left(x^{*}\right)<f(\bar{x}) .
$$

By the explicit $(\alpha, m)$-preinvexity of $f(x)$ and the fact that $m\left(1-\lambda^{\alpha}\right)+\lambda^{\alpha} \leq 1$, we can deduce that

$$
f\left(m \bar{x}+\lambda \eta\left(x^{*}, \bar{x}, m\right)\right)<m\left(1-\lambda^{\alpha}\right) f(\bar{x})+\lambda^{\alpha} f\left(x^{*}\right)<\left[m\left(1-\lambda^{\alpha}\right)+\lambda^{\alpha}\right] f(\bar{x})<f(\bar{x})
$$

for all $0<\lambda<1$. For a sufficiently small $\lambda>0$, it follows that

$$
m \bar{x}+\lambda \eta\left(x^{*}, \bar{x}, m\right) \in K \cap N_{\varepsilon}(\bar{x})
$$

which is a contradiction to (2.5). This completes the proof.

By Proposition 2.9, we can conclude that explicitly $(\alpha, m)$-preinvex functions constitute an important class of generalized convex functions in mathematical programming. The function in Example 2.1 is not an explicitly $(\alpha, m)$-preinvex function with respect to $\eta$ based on Proposition 2.9.

For investigating the relationship between the generalized $(\alpha, m)$-preinvex function and the generalized quasi $m$-preinvex function, we will present the extended Condition $C$ and Lemma 2.1.

Let us recall the Condition C introduced by Mohan and Neogy [39] as follows.

Condition C: Let $\eta: \mathbb{R}^{n} \times \mathbb{R}^{n} \rightarrow \mathbb{R}^{n}$, we say that the mapping $\eta$ satisfies the condition $\mathrm{C}$ if for any $x, y \in \mathbb{R}^{n}$,

$\left(\mathrm{C}_{1}\right) \eta(x, x+\lambda \eta(y, x))=-\lambda \eta(y, x)$

$\left(C_{2}\right) \eta(y, x+\lambda \eta(y, x))=(1-\lambda) \eta(y, x)$,

for all $\lambda \in[0,1]$, hold.

Similarly, we present here the so-called 'extended Condition C'.

Extended Condition C: Let $\eta: \mathbb{R}^{n} \times \mathbb{R}^{n} \times(0,1] \rightarrow \mathbb{R}^{n}$, we say that the mapping $\eta$ satisfies the extended condition $C$ if for any $x, y \in \mathbb{R}^{n}$,

$\left(\mathrm{C}_{1}\right) \eta(x, m x+\lambda \eta(y, x, m), m)=-\lambda \eta(y, x, m)$

$\left(C_{2}\right) \eta(y, m x+\lambda \eta(y, x, m), m)=(1-\lambda) \eta(y, x, m)$,

$\left(\mathrm{C}_{3}\right) \eta(y, x, m)=-\eta(x, y, m)$,

for all $\lambda \in[0,1]$ and fixed $m \in(0,1]$, hold.

Lemma 2.1 Let $K \subseteq \mathbb{R}^{n}$ be a nonempty m-invex set with respect to the mapping $\eta: \mathbb{R}^{n} \times$ $\mathbb{R}^{n} \times(0,1] \rightarrow \mathbb{R}^{n}$ and $\eta$ satisfies the extended Condition $C$. If $: K \rightarrow \mathbb{R}_{0}$ satisfies $f(m x+$ 
$\eta(y, x, m)) \leq f(y), \forall x, y \in K$, and there exists a $t \in(0,1)$ such that

$$
f(m x+t \eta(y, x, m)) \leq m\left(1-t^{\alpha}\right) f(x)+t^{\alpha} f(y), \quad \forall x, y \in K
$$

then the set $A=\left\{\lambda \in[0,1] \mid f(m x+\lambda \eta(y, x, m)) \leq m\left(1-\lambda^{\alpha}\right) f(x)+\lambda^{\alpha} f(y), \forall x, y \in K\right\}$ is dense in $[0,1]$.

The proof of Lemma 2.1 is much akin to that of given method for Lemma 3.2 in [40], p.232. The details are left to the interested reader. The next theorem shows the relationship between the generalized $(\alpha, m)$-preinvex function and the generalized quasi $m$-preinvex function.

Theorem 2.1 Let $K$ be a nonempty $m$-invex set in $\mathbb{R}_{0}$ with respect to $\eta: \mathbb{R} \times \mathbb{R} \times(0,1] \rightarrow$ $\mathbb{R}$, where $\eta$ satisfies the extended Condition $C$. Then the real-value decrease function $f$ : $K \rightarrow \mathbb{R}_{0}$ is a generalized $(\alpha, m)$-preinvex function if and only if it is a generalized quasi $m$-preinvex function on $K$ and there exists a $t \in(0,1)$ such that

$$
f(m x+t \eta(y, x, m)) \leq m\left(1-t^{\alpha}\right) f(x)+t^{\alpha} f(y), \quad \forall x, y \in K
$$

Proof The necessity is proofed by Proposition 2.1. We only need prove the sufficiency.

For every $x, y \in K$, let $z_{\lambda}=m x+\lambda \eta(y, x, m), \lambda \in[0,1]$. Two different situations where $m f(x)=f(y)$ or $m f(x) \neq f(y)$ will be considered as follows, respectively.

(I) $m f(x)=f(y)$. We need to prove that

$$
f(m x+\lambda \eta(y, x, m)) \leq m\left(1-\lambda^{\alpha}\right) f(x)+\lambda^{\alpha} f(y), \quad \forall \lambda \in[0,1]
$$

By contradiction, assume that there exists $\beta \in(0,1]$ such that

$$
f\left(z_{\beta}\right)=f(m x+\beta \eta(y, x, m))>m\left(1-\beta^{\alpha}\right) f(x)+\beta^{\alpha} f(y)=m f(x)=f(y)
$$

(i) Suppose that $0<\gamma<\beta \leq 1$. Let $\mu=\frac{\beta-\gamma}{1-\gamma}$. From the extended Condition $C$, we have

$$
z_{\beta}=z_{\mu}+\gamma \eta\left(y, z_{\mu}, m\right)
$$

From (2.7) and (2.8) and the decrease of $f$ on $K$, we deduce that

$$
\begin{aligned}
f\left(z_{\beta}\right) & =f\left(z_{\mu}+\gamma \eta\left(y, z_{\mu}, m\right)\right) \\
& \leq f\left(m z_{\mu}+\gamma \eta\left(y, z_{\mu}, m\right)\right) \\
& \leq m\left(1-\gamma^{\alpha}\right) f\left(z_{\mu}\right)+\gamma^{\alpha} f(y) \\
& <m f\left(z_{\mu}\right) \\
& <f\left(z_{\mu}\right) .
\end{aligned}
$$

To prove the third inequality above, we used the fact that $f(y)<m f\left(z_{\mu}\right)$. Otherwise, this breeds a contradiction to (2.8). On the other hand, let $\delta=\frac{\beta-\mu}{\beta}$ and from the extended 
Condition $\mathrm{C}$, we get

$$
z_{\mu}=z_{\beta}+\delta \eta\left(x, z_{\beta}, m\right)
$$

Consequently, from the decrease of $f$ on $K$ and the generalized quasi $m$-preinvexity of $f$, we derive that

$$
f\left(z_{\mu}\right)=f\left(z_{\beta}+\delta \eta\left(x, z_{\beta}, m\right)\right) \leq f\left(m z_{\beta}+\delta \eta\left(x, z_{\beta}, m\right)\right) \leq \max \left\{f\left(z_{\beta}\right), f(x)\right\} .
$$

(a) If $f(x) \leq f\left(z_{\beta}\right)$, from the inequality (2.10), we have $f\left(z_{\mu}\right) \leq f\left(z_{\beta}\right)$, which contradicts the inequality (2.9).

(b) If $f(x)>f\left(z_{\beta}\right)$, from the inequality (2.10), we have $f\left(z_{\mu}\right) \leq f(x)$, which contradicts the fact that $f(x)<f\left(z_{\mu}\right)$.

(ii) Assume that $0<\beta<\gamma \leq 1$. Let $\mu=\frac{\beta}{\gamma}>\beta$. From the extended Condition C, we obtain

$$
z_{\beta}=m x+\gamma \eta\left(z_{\mu}, x, m\right)
$$

From (2.7) and (2.11) as well as (2.8), we deduce that

$$
f\left(z_{\beta}\right)=f\left(m x+\gamma \eta\left(z_{\mu}, x, m\right)\right) \leq m\left(1-\gamma^{\alpha}\right) f(x)+\gamma^{\alpha} f\left(z_{\mu}\right)<f\left(z_{\mu}\right) .
$$

Let $\delta=\frac{\mu-\beta}{1-\beta}$, by the extended Condition $C$, we have

$$
z_{\mu}=z_{\beta}+\delta \eta\left(y, z_{\beta}, m\right)
$$

In the same way, from (2.7) and (2.13) as well as (2.8), we get

$$
\begin{aligned}
f\left(z_{\mu}\right) & =f\left(z_{\beta}+\delta \eta\left(y, z_{\beta}, m\right)\right) \\
& \leq f\left(m z_{\beta}+\delta \eta\left(y, z_{\beta}, m\right)\right) \\
& \leq m\left(1-\gamma^{\alpha}\right) f\left(z_{\beta}\right)+\gamma^{\alpha} f(y) \\
& <f\left(z_{\beta}\right) .
\end{aligned}
$$

which contradicts the inequality (2.12).

(II) $m f(x) \neq f(y)$. In this case, we also need to prove that

$$
f(m x+\lambda \eta(y, x, m)) \leq m\left(1-\lambda^{\alpha}\right) f(x)+\lambda^{\alpha} f(y), \quad \forall \lambda \in[0,1] .
$$

By contradiction, assume that there exists $\beta \in(0,1)$ such that

$$
f\left(z_{\beta}\right)=f(m x+\beta \eta(y, x, m))>m\left(1-\beta^{\alpha}\right) f(x)+\beta^{\alpha} f(y) .
$$

From Lemma 2.1, we know that, for $A$, defined in Lemma 2.1,

$$
f(m x+\lambda \eta(y, x, m)) \leq m\left(1-\lambda^{\alpha}\right) f(x)+\lambda^{\alpha} f(y), \quad \forall \lambda \in A .
$$


(1) Assume that $m f(x)>f(y)$. Then from (2.14) and the density of $A$, there exists $\mu \in A$ with $\mu<\beta$ such that

$$
\begin{aligned}
f\left(z_{\mu}\right) & =f(m x+\mu \eta(y, x, m)) \\
& \leq m\left(1-\mu^{\alpha}\right) f(x)+\mu^{\alpha} f(y) \\
& \leq f(m x+\beta \eta(y, x, m)) \\
& =f\left(z_{\beta}\right) .
\end{aligned}
$$

Let $\delta=\frac{\beta-\mu}{1-\mu}$. Clearly $0<\delta<1$ and from the extended Condition $\mathrm{C}$, we have

$$
z_{\beta}=z_{\mu}+\delta \eta\left(y, z_{\mu}, m\right)
$$

(a) If $f(y) \leq f\left(z_{\mu}\right)$, from the decrease generalized quasi-m-preinvexity of $f$, we obtain

$$
\begin{aligned}
f\left(z_{\beta}\right) & =f\left(z_{\mu}+\delta \eta\left(y, z_{\mu}, m\right)\right) \\
& \leq f\left(m z_{\mu}+\delta \eta\left(y, z_{\mu}, m\right)\right) \\
& \leq \max \left\{f\left(z_{\mu}\right), f(y)\right\} \\
& \leq f\left(z_{\mu}\right),
\end{aligned}
$$

which contradicts the inequality (2.15).

(b) If $f(y)>f\left(z_{\mu}\right)$, similarly, by the decrease generalized quasi-m-preinvexity of $f$ and $m f(x)>f(y)$ we obtain

$$
\begin{aligned}
f\left(z_{\beta}\right) & =f\left(z_{\mu}+\delta \eta\left(y, z_{\mu}, m\right)\right) \\
& \leq f\left(m z_{\mu}+\delta \eta\left(y, z_{\mu}, m\right)\right) \\
& \leq \max \left\{f\left(z_{\mu}\right), f(y)\right\} \\
& \leq f(y) \\
& <m\left(1-\beta^{\alpha}\right) f(x)+\beta^{\alpha} f(y) \\
& <f\left(z_{\beta}\right),
\end{aligned}
$$

which is a contradiction.

(2) Assume that $m f(x)<f(y)$. Then from (2.14) and the density of $A$, there exists $\mu \in A$ with $\mu>\beta$ such that

$$
\begin{aligned}
f\left(z_{\mu}\right) & =f(m x+\mu \eta(y, x, m)) \\
& \leq m\left(1-\mu^{\alpha}\right) f(x)+\mu^{\alpha} f(y) \\
& \leq f(m x+\beta \eta(y, x, m)) \\
& =f\left(z_{\beta}\right) .
\end{aligned}
$$

Let $\delta=\frac{\beta}{\mu}$. Obviously $0<\delta<1$ and from the extended Condition $\mathrm{C}$, we have

$$
z_{\beta}=m x+\delta \eta\left(z_{\mu}, x, m\right)
$$


(a) If $m f(x) \leq f\left(z_{\mu}\right)$, from (2.7) and (2.17), we obtain

$$
f\left(z_{\beta}\right)=f\left(m x+\delta \eta\left(z_{\mu}, x, m\right)\right) \leq m\left(1-\delta^{\alpha}\right) f(x)+\delta^{\alpha} f\left(z_{\mu}\right) \leq f\left(z_{\mu}\right)
$$

which contradicts the inequality (2.16).

(b) If $m f(x)>f\left(z_{\mu}\right)$, in the same way, and utilizing $m f(x)<f(y)$, we obtain

$$
\begin{aligned}
f\left(z_{\beta}\right) & =f\left(m x+\delta \eta\left(z_{\mu}, x, m\right)\right) \\
& \leq m\left(1-\delta^{\alpha}\right) f(x)+\delta^{\alpha} f\left(z_{\mu}\right) \\
& \leq m f(x) \\
& <m\left(1-\beta^{\alpha}\right) f(x)+\beta^{\alpha} f(y) \\
& <f\left(z_{\beta}\right),
\end{aligned}
$$

which is a contradiction. This completes the proof.

The result established by Theorem 2.1 shows that under certain conditions the generalized $(\alpha, m)$-preinvexity is equivalent to the generalized quasi- $m$-preinvexity when there exists a point to satisfy generalized $(\alpha, m)$-preinvexity. The extended Condition $\mathrm{C}$ seems to be an indispensable hypothesis.

\section{Riemann-Liouville fractional Hermite-Hadamard inequalities}

Let $f: K \rightarrow \mathbb{R}$ be a differentiable function, throughout this section we will take

$$
\begin{aligned}
R_{f}(\alpha ; \eta, m, a, b):= & \frac{f(m a)+f(m a+\eta(b, a, m))}{2} \\
& -\frac{\Gamma(\alpha+1)}{2 \eta^{\alpha}(b, a, m)}\left[J_{m a^{+}}^{\alpha} f(m a+\eta(b, a, m))+J_{(m a+\eta(b, a, m))^{-}}^{\alpha} f(m a)\right],
\end{aligned}
$$

where $K \subseteq \mathbb{R}$ be an open $m$-invex subset with respect to $\eta: K \times K \times(0,1] \rightarrow \mathbb{R}$ for some fixed $m \in(0,1], a, b \in K$ with $a<b, \alpha>0$ and $\Gamma$ is the Euler Gamma function.

We prove the following lemma to obtain our new results in this section.

Lemma 3.1 Let $K \subseteq \mathbb{R}$ be an open m-invex subset with respect to $\eta: K \times K \times(0,1] \rightarrow \mathbb{R}$ for some fixed $m \in(0,1]$ and let $a, b \in K, a<b$ with $\eta(b, a, m)>0$. Assume that $f: K \rightarrow \mathbb{R}$ is a twice differentiable function, $f^{\prime \prime}$ is integrable on $[m a, m a+\eta(b, a, m)]$, then the following identity for the Riemann-Louville fractional integral with $\alpha>0$ and $x \in[m a, m a+$ $\eta(b, a, m)]$ holds:

$$
R_{f}(\alpha ; \eta, m, a, b)=\frac{\eta^{2}(b, a, m)}{2} \int_{0}^{1} \frac{1-t^{\alpha+1}-(1-t)^{\alpha+1}}{\alpha+1} f^{\prime \prime}(m a+t \eta(b, a, m)) \mathrm{d} t
$$

Proof Set

$$
I=\frac{\eta^{2}(b, a, m)}{2} \int_{0}^{1} \frac{1-t^{\alpha+1}-(1-t)^{\alpha+1}}{\alpha+1} f^{\prime \prime}(m a+t \eta(b, a, m)) \mathrm{d} t .
$$


Since $a, b \in K$ and $K$ is an $m$-invex subset with respect to $\eta$, for every $t \in[0,1]$ and some fixed $m \in(0,1]$, we have $m a+t \eta(b, a, m) \in K$. Integrating by part yields

$$
\begin{aligned}
I= & \frac{\eta^{2}(b, a, m)}{2}\left[\left.\frac{1-t^{\alpha+1}-(1-t)^{\alpha+1}}{(\alpha+1) \eta(b, a, m)} f^{\prime}(m a+t \eta(b, a, m))\right|_{0} ^{1}\right. \\
& \left.-\int_{0}^{1} \frac{-(\alpha+1) t^{\alpha}+(1-t)^{\alpha}(\alpha+1)}{(\alpha+1) \eta(b, a, m)} f^{\prime}(m a+t \eta(b, a, m)) \mathrm{d} t\right] \\
= & \frac{\eta^{2}(b, a, m)}{2}\left[\frac{f(m a+\eta(b, a, m))}{\eta^{2}(b, a, m)}+\frac{f(m a)}{\eta^{2}(b, a, m)}\right. \\
& \left.-\int_{0}^{1} \frac{\alpha t^{\alpha-1}+\alpha(1-t)^{\alpha-1}}{\eta^{2}(b, a, m)} f(m a+t \eta(b, a, m)) \mathrm{d} t\right] \\
= & \frac{f(m a)+f(m a+\eta(b, a, m))}{2} \\
& -\frac{\alpha}{2}\left[\int_{0}^{1}\left(t^{\alpha-1}+(1-t)^{\alpha-1}\right) f(m a+t \eta(b, a, m)) \mathrm{d} t\right] .
\end{aligned}
$$

Let $u=m a+t \eta(b, a, m)$, then $\mathrm{d} u=\eta(b, a, m) \mathrm{d} t$, and using the reduction formula $\Gamma(\alpha+1)=$ $\alpha \Gamma(\alpha)(\alpha>0)$ for Euler Gamma function, we have

$$
\frac{\alpha}{2}\left[\int_{0}^{1} t^{\alpha-1} f(m a+t \eta(b, a, m)) \mathrm{d} t\right]=\frac{\Gamma(\alpha+1)}{2 \eta^{\alpha}(b, a, m)} J_{(m a+\eta(b, a, m))}^{\alpha} f(m a)
$$

and similarly we get

$$
\frac{\alpha}{2}\left[\int_{0}^{1}(1-t)^{\alpha-1} f(m a+t \eta(b, a, m)) \mathrm{d} t\right]=\frac{\Gamma(\alpha+1)}{2 \eta^{\alpha}(b, a, m)} J_{m a^{+}}^{\alpha} f(m a+\eta(b, a, m) .
$$

Thus, we have conclusion (3.1).

Remark 3.1 If $\eta(b, a, m)=b-m a$ with $m=1$ in Lemma 3.1, then the identity (3.1) reduces to the following identity:

$$
\begin{aligned}
& \frac{f(a)+f(b)}{2}-\frac{\Gamma(\alpha+1)}{2(b-a)^{\alpha}}\left[J_{a^{+}}^{\alpha} f(b)+J_{b^{-}}^{\alpha} f(a)\right] \\
& =\frac{(b-a)^{2}}{2} \int_{0}^{1} \frac{1-t^{\alpha+1}-(1-t)^{\alpha+1}}{\alpha+1} f^{\prime \prime}(t b+(1-t) a) \mathrm{d} t .
\end{aligned}
$$

By using $J_{b^{+}}^{\alpha} f(a)+J_{a^{-}}^{\alpha} f(b)=(-1)^{\alpha}\left[J_{a^{+}}^{\alpha} f(b)+J_{b^{-}}^{\alpha} f(a)\right]$ and exchanging $a$ with $b$ in (3.2), it follows that

$$
\begin{aligned}
& \frac{f(a)+f(b)}{2}-\frac{\Gamma(\alpha+1)}{2(b-a)^{\alpha}}\left[J_{a^{+}}^{\alpha} f(b)+J_{b^{-}}^{\alpha} f(a)\right] \\
& =\frac{(b-a)^{2}}{2} \int_{0}^{1} \frac{1-t^{\alpha+1}-(1-t)^{\alpha+1}}{\alpha+1} f^{\prime \prime}(t a+(1-t) b) \mathrm{d} t,
\end{aligned}
$$

which is proved by Wang et al. [30]. Based on this identity, they established some interesting Riemann-Liouville fractional integrals for $m$-convex and $(s, m)$-convex mappings, respectively. 
If we choose $\alpha=1$ in (3.3), it follows that

$$
\frac{f(a)+f(b)}{2}-\frac{1}{b-a} \int_{a}^{b} f(t) \mathrm{d} t=\frac{(b-a)^{2}}{2} \int_{0}^{1} t(1-t) f^{\prime \prime}(t a+(1-t) b) \mathrm{d} t
$$

which is used by Ödemir, Avci and Set in [6] to establish many interesting HermiteHadamard-type inequalities for $m$-convexity.

With the help of Lemma 3.1, new upper bound for the right-hand side of (1.6) for generalized $(\alpha, m)$-preinvex functions via the Riemann-Liouville fractional integral is presented in the following theorem.

Theorem 3.1 Let $A \subseteq \mathbb{R}$ be an open m-invex subset with respect to $\eta: A \times A \times(0,1] \rightarrow \mathbb{R}$ for some fixed $m \in(0,1]$ and let $a, b \in A, a<b$ with $\eta(b, a, m)>0$. Assume that $f: A \rightarrow \mathbb{R}$ is a twice differentiable function, $\left|f^{\prime \prime}\right|$ is a generalized $(\alpha, m)$-preinvex function on $A$ for some fixed $\alpha, m \in(0,1]$ and $x \in[m a, m a+\eta(b, a, m)]$, then the following inequality for the Riemann-Louville fractional integral with $0<\alpha \leq 1$ holds:

$$
\begin{aligned}
& \left|R_{f}(\alpha ; \eta, m, a, b)\right| \\
& \quad \leq \frac{\eta^{2}(b, a, m)}{2(\alpha+1)}\left[m\left(\frac{2 \alpha^{2}+\alpha-2}{(\alpha+2)(2 \alpha+2)}+\beta(\alpha+1, \alpha+2)\right)\left|f^{\prime \prime}(a)\right|\right. \\
& \left.\quad+\left(\frac{1}{2 \alpha+2}-\beta(\alpha+1, \alpha+2)\right)\left|f^{\prime \prime}(b)\right|\right] .
\end{aligned}
$$

Proof Since $m a+t \eta(b, a, m) \in A$ for each $t \in[0,1]$, by using the properties of modulus on Lemma 3.1, we can obtain

$$
\left|R_{f}(\alpha ; \eta, m, a, b)\right| \leq \frac{\eta^{2}(b, a, m)}{2} \int_{0}^{1}\left|\frac{1-t^{\alpha+1}-(1-t)^{\alpha+1}}{\alpha+1}\right|\left|f^{\prime \prime}(m a+t \eta(b, a, m))\right| \mathrm{d} t
$$

Using the generalized $(\alpha, m)$-preinvexity of $\left|f^{\prime \prime}\right|$ on $A$, we have

$$
\begin{aligned}
\int_{0}^{1} & \frac{1-t^{\alpha+1}-(1-t)^{\alpha+1}}{\alpha+1}\left|f^{\prime \prime}(m a+t \eta(b, a, m))\right| \mathrm{d} t \\
\leq & \frac{1}{\alpha+1} \int_{0}^{1}\left(1-t^{\alpha+1}-(1-t)^{\alpha+1}\right)\left(m\left(1-t^{\alpha}\right)\left|f^{\prime \prime}(a)\right|+t^{\alpha}\left|f^{\prime \prime}(b)\right|\right) \mathrm{d} t \\
\leq & \frac{1}{\alpha+1}\left[m\left(\frac{2 \alpha^{2}+\alpha-2}{(\alpha+2)(2 \alpha+2)}+\beta(\alpha+1, \alpha+2)\right)\left|f^{\prime \prime}(a)\right|\right. \\
& \left.+\left(\frac{1}{2 \alpha+2}-\beta(\alpha+1, \alpha+2)\right)\left|f^{\prime \prime}(b)\right|\right] .
\end{aligned}
$$

To prove the second inequality above, we used the facts that

$$
\begin{aligned}
& \int_{0}^{1}\left(1-t^{\alpha+1}-(1-t)^{\alpha+1}-t^{\alpha}+t^{2 \alpha+1}\right) \mathrm{d} t=\frac{2 \alpha^{2}+\alpha-2}{(\alpha+2)(2 \alpha+2)} \\
& \int_{0}^{1}\left(t^{\alpha}-t^{2 \alpha+1}\right) \mathrm{d} t=\frac{1}{2 \alpha+2}
\end{aligned}
$$


and

$$
\int_{0}^{1} t^{\alpha}(1-t)^{\alpha+1} \mathrm{~d} t=\beta(\alpha+1, \alpha+2)
$$

where the Beta function,

$$
\beta(x, y)=\int_{0}^{1} t^{x-1}(1-t)^{y-1} \mathrm{~d} t, \quad \forall x, y>0
$$

which completes the proof.

By means of elementary calculation, it is easy to deduce the following results.

Corollary 3.1 With the same assumptions given in Theorem 3.1, if $\eta(b, a, m)=b-m a$, we obtain

$$
\begin{aligned}
& \left|\frac{f(m a)+f(b)}{2}-\frac{\Gamma(\alpha+1)}{2(b-m a)^{\alpha}}\left[J_{m a^{+}}^{\alpha} f(b)+J_{b^{-}}^{\alpha} f(m a)\right]\right| \\
& \leq \frac{(b-m a)^{2}}{2(\alpha+1)}\left[m\left(\frac{2 \alpha^{2}+\alpha-2}{(\alpha+2)(2 \alpha+2)}+\beta(\alpha+1, \alpha+2)\right)\left|f^{\prime \prime}(a)\right|\right. \\
& \left.\quad+\left(\frac{1}{2 \alpha+2}-\beta(\alpha+1, \alpha+2)\right)\left|f^{\prime \prime}(b)\right|\right]
\end{aligned}
$$

specially for $\alpha=m=1$, we get

$$
\left|\frac{f(a)+f(b)}{2}-\frac{1}{b-a} \int_{a}^{b} f(x) \mathrm{d} x\right| \leq \frac{(b-a)^{2}}{12}\left[\frac{\left|f^{\prime \prime}(a)\right|+\left|f^{\prime \prime}(b)\right|}{2}\right] .
$$

This is one of the inequalities given in [38], Theorem 2.

Corollary 3.2 In Theorem 3.1, if the mapping $\eta(b, a, m)$ with $m=1$ degenerates into $\eta(b, a)$ and we choose $\alpha=1$, then (3.4) becomes

$$
\left|\frac{f(a)+f(a+\eta(b, a))}{2}-\frac{1}{\eta(b, a)} \int_{a}^{a+\eta(b, a)} f(x) \mathrm{d} x\right| \leq \frac{\eta^{2}(b, a)}{24}\left[\left|f^{\prime \prime}(a)\right|+\left|f^{\prime \prime}(b)\right|\right],
$$

which is the same as the inequality established in [37], Theorem 4.1.

Theorem 3.2 Let fbe defined as in Theorem 3.1, If the function $\left|f^{\prime \prime}\right|^{q}$ for $q>1$ is a generalized $(\alpha, m)$-preinvex function on A for some fixed $\alpha, m \in(0,1]$ and $x \in[m a, m a+\eta(b, a, m)]$, then the following inequality for the Riemann-Louville fractional integral with $0<\alpha \leq 1$ holds:

$$
\begin{aligned}
& \left|R_{f}(\alpha ; \eta, m, a, b)\right| \\
& \leq \leq \frac{\eta^{2}(b, a, m)}{2(\alpha+1)}\left\{m\left[\frac{q \alpha+\alpha+1}{(\alpha+1)(q+1)}-\frac{2}{q(\alpha+1)+1}+\beta(\alpha+1, q(\alpha+1)+1)\right]\left|f^{\prime \prime}(a)\right|^{q}\right. \\
& \left.\quad+\left[\frac{q}{(\alpha+1)(q+1)}-\beta(\alpha+1, q(\alpha+1)+1)\right]\left|f^{\prime \prime}(b)\right|^{q}\right\}^{\frac{1}{q}} .
\end{aligned}
$$


Proof Since $m a+t \eta(b, a, m) \in A$ for every $t \in[0,1]$, by using the properties of modulus on Lemma 3.1 and making use of Hölder's integral inequality for $q>1$, we can obtain

$$
\begin{aligned}
& \left|R_{f}(\alpha ; \eta, m, a, b)\right| \\
& \quad \leq \frac{\eta^{2}(b, a, m)}{2} \int_{0}^{1}\left|\frac{1-t^{\alpha+1}-(1-t)^{\alpha+1}}{\alpha+1}\right| f^{\prime \prime}(m a+t \eta(b, a, m)) \mid \mathrm{d} t \\
& \quad \leq \frac{\eta^{2}(b, a, m)}{2(\alpha+1)}\left(\int_{0}^{1} 1 \mathrm{~d} t\right)^{1-\frac{1}{q}}\left[\int_{0}^{1}\left(1-t^{\alpha+1}-(1-t)^{\alpha+1}\right)^{q}\left|f^{\prime \prime}(m a+t \eta(b, a, m))\right|^{q} \mathrm{~d} t\right]^{\frac{1}{q}} \\
& \quad \leq \frac{\eta^{2}(b, a, m)}{2(\alpha+1)}\left[\int_{0}^{1}\left(1-t^{q(\alpha+1)}-(1-t)^{q(\alpha+1)}\right)\left|f^{\prime \prime}(m a+t \eta(b, a, m))\right|^{q} \mathrm{~d} t\right]^{\frac{1}{q}} .
\end{aligned}
$$

To prove the third inequality above, we used the following inequality:

$$
\left(1-t^{\alpha+1}-(1-t)^{\alpha+1}\right)^{q} \leq 1-t^{q(\alpha+1)}-(1-t)^{q(\alpha+1)}
$$

for any $t \in[0,1]$, which follows from

$$
(A-B)^{q} \leq A^{q}-B^{q}
$$

for any $A>B \geq 0$ and $q \geq 1$.

Using the generalized $(\alpha, m)$-preinvexity of $\left|f^{\prime \prime}\right|^{q}$ on $A$, we have

$$
\begin{aligned}
& \int_{0}^{1}\left(1-t^{q(\alpha+1)}-(1-t)^{q(\alpha+1)}\right)\left|f^{\prime \prime}(m a+t \eta(b, a, m))\right|^{q} \mathrm{~d} t \\
& \quad \leq \int_{0}^{1}\left(1-t^{(\alpha+1) q}-(1-t)^{(\alpha+1) q}\right)\left(m\left(1-t^{\alpha}\right)\left|f^{\prime \prime}(a)\right|^{q}+t^{\alpha}\left|f^{\prime \prime}(b)\right|^{q}\right) \mathrm{d} t \\
& =m\left[\frac{\alpha q+\alpha+1}{(\alpha+1)(q+1)}-\frac{2}{q(\alpha+1)+1}+\beta(\alpha+1, q(\alpha+1)+1)\right]\left|f^{\prime \prime}(a)\right|^{q} \\
& \quad+\left[\frac{q}{(\alpha+1)(q+1)}-\beta(\alpha+1, q(\alpha+1)+1)\right]\left|f^{\prime \prime}(b)\right|^{q} .
\end{aligned}
$$

Thus, we can get the desired result.

Direct computation yields the following corollary.

Corollary 3.3 With the same assumptions given in Theorem 3.2, if $\eta(b, a, m)=b-m a$, we obtain

$$
\begin{aligned}
& \left|\frac{f(m a)+f(b)}{2}-\frac{\Gamma(\alpha+1)}{2(b-m a)^{\alpha}}\left[J_{m a^{+}}^{\alpha} f(b)+J_{b^{-}}^{\alpha} f(m a)\right]\right| \\
& \leq \frac{(b-m a)^{2}}{2(\alpha+1)}\left\{m\left[\frac{q \alpha+\alpha+1}{(\alpha+1)(q+1)}-\frac{2}{q(\alpha+1)+1}+\beta(\alpha+1, q(\alpha+1)+1)\right]\left|f^{\prime \prime}(a)\right|^{q}\right. \\
& \left.\quad+\left[\frac{q}{(\alpha+1)(q+1)}-\beta(\alpha+1, q(\alpha+1)+1)\right]\left|f^{\prime \prime}(b)\right|^{q}\right\}^{\frac{1}{q}} ;
\end{aligned}
$$


specially for $\alpha=m=1$ and $\left|f^{\prime \prime}\right| \leq K$ on $[a, b]$, we get

$$
\begin{aligned}
\left|\frac{f(a)+f(b)}{2}-\frac{1}{b-a} \int_{a}^{b} f(x) \mathrm{d} x\right| & \leq \frac{(b-a)^{2}}{4}\left(\frac{2 q-1}{2 q+1}\right)^{\frac{1}{q}} K \\
& \leq \frac{(b-a)^{2}}{4} K .
\end{aligned}
$$

For proving the second inequality of (3.7), we use the facts that

$$
\lim _{q \rightarrow 1^{+}}\left(\frac{2 q-1}{2 q+1}\right)^{\frac{1}{q}}=\frac{1}{3}
$$

and

$$
\lim _{q \rightarrow \infty}\left(\frac{2 q-1}{2 q+1}\right)^{\frac{1}{q}}=1
$$

Therefore, we have

$$
\frac{1}{3}<\left(\frac{2 q-1}{2 q+1}\right)^{\frac{1}{q}}<1, \quad q \in(1, \infty)
$$

A similar result is presented in the following theorem.

Theorem 3.3 Letfbe defined as in Theorem 3.1 with $\frac{1}{p}+\frac{1}{q}=1, q>1$. If $\left|f^{\prime \prime}\right|^{q}$ is a generalized $(\alpha, m)$-preinvex function on A for some fixed $\alpha, m \in(0,1]$ and $x \in[m a, m a+\eta(b, a, m)]$, then the following inequality for the Riemann-Louville fractional integral with $0<\alpha \leq 1$ holds:

$$
\begin{aligned}
& \left|R_{f}(\alpha ; \eta, m, a, b)\right| \\
& \quad \leq \frac{\eta^{2}(b, a, m)}{2(\alpha+1)}\left(\frac{p \alpha+p-1}{p \alpha+p+1}\right)^{\frac{1}{p}}\left(\frac{m \alpha}{\alpha+1}\left|f^{\prime \prime}(a)\right|^{q}+\frac{1}{\alpha+1}\left|f^{\prime \prime}(b)\right|^{q}\right)^{\frac{1}{q}} .
\end{aligned}
$$

Proof Since $m a+t \eta(b, a, m) \in A$ for every $t \in[0,1]$, by using the properties of modulus on Lemma 3.1 and Hölder's integral inequality for $q>1$, we can obtain

$$
\begin{aligned}
& \left|R_{f}(\alpha ; \eta, m, a, b)\right| \\
& \quad \leq \frac{\eta^{2}(b, a, m)}{2} \int_{0}^{1}\left|\frac{1-t^{\alpha+1}-(1-t)^{\alpha+1}}{\alpha+1}\right|\left|f^{\prime \prime}(m a+t \eta(b, a, m))\right| \mathrm{d} t \\
& \quad \leq \frac{\eta^{2}(b, a, m)}{2(\alpha+1)}\left(\int_{0}^{1}\left|1-t^{\alpha+1}-(1-t)^{\alpha+1}\right|^{p} \mathrm{~d} t\right)^{\frac{1}{p}}\left(\int_{0}^{1}\left|f^{\prime \prime}(m a+t \eta(b, a, m))\right|^{q} \mathrm{~d} t\right)^{\frac{1}{q}} .
\end{aligned}
$$

Using the inequality (3.6) and the generalized $(\alpha, m)$-preinvexity of $\left|f^{\prime \prime}\right|^{q}$ on $A$, we have

$$
\begin{aligned}
& \left|R_{f}(\alpha ; \eta, m, a, b)\right| \\
& \quad \leq \frac{\eta^{2}(b, a, m)}{2(\alpha+1)}\left(\int_{0}^{1}\left(1-t^{p(\alpha+1)}-(1-t)^{p(\alpha+1)}\right) \mathrm{d} t\right)^{\frac{1}{p}}
\end{aligned}
$$




$$
\begin{aligned}
& \times\left(\int_{0}^{1}\left(m\left(1-t^{\alpha}\right)\left|f^{\prime \prime}(a)\right|^{q}+t^{\alpha}\left|f^{\prime \prime}(b)\right|^{q}\right) \mathrm{d} t\right)^{\frac{1}{q}} \\
= & \frac{\eta^{2}(b, a, m)}{2(\alpha+1)}\left(\frac{p \alpha+p-1}{p \alpha+p+1}\right)^{\frac{1}{p}}\left(\frac{m \alpha}{\alpha+1}\left|f^{\prime \prime}(a)\right|^{q}+\frac{1}{\alpha+1}\left|f^{\prime \prime}(b)\right|^{q}\right)^{\frac{1}{q}} .
\end{aligned}
$$

Therefore, we can get the required results.

Elementary calculation provides the following corollaries.

Corollary 3.4 With the same assumptions given in Theorem 3.3, if $\eta(b, a, m)=b-m a$, we obtain

$$
\begin{aligned}
& \left|\frac{f(m a)+f(b)}{2}-\frac{\Gamma(\alpha+1)}{2(b-m a)^{\alpha}}\left[J_{m a^{+}}^{\alpha} f(b)+J_{b^{-}}^{\alpha} f(m a)\right]\right| \\
& \quad \leq \frac{(b-m a)^{2}}{2(\alpha+1)}\left(\frac{p \alpha+p-1}{p \alpha+p+1}\right)^{\frac{1}{p}}\left(\frac{m \alpha}{\alpha+1}\left|f^{\prime \prime}(a)\right|^{q}+\frac{1}{\alpha+1}\left|f^{\prime \prime}(b)\right|^{q}\right)^{\frac{1}{q}},
\end{aligned}
$$

specially for $\alpha=m=1$ and $\left|f^{\prime \prime}\right| \leq K$ on $[a, b]$, we get

$$
\left|\frac{f(a)+f(b)}{2}-\frac{1}{b-a} \int_{a}^{b} f(x) \mathrm{d} x\right| \leq \frac{(b-a)^{2}}{4}\left(\frac{2 p-1}{2 p+1}\right)^{\frac{1}{p}} K .
$$

Corollary 3.5 In Theorem 3.3 , if the mapping $\eta(b, a, m)$ with $m=1$ degenerates into $\eta(b, a)$ and we choose $\alpha=1$, then (3.9) becomes

$$
\begin{aligned}
& \left|\frac{f(a)+f(a+\eta(b, a))}{2}-\frac{1}{\eta(b, a)} \int_{a}^{a+\eta(b, a)} f(x) \mathrm{d} x\right| \\
& \leq \frac{\eta^{2}(b-a)}{4}\left(\frac{2 p-1}{2 p+1}\right)^{\frac{1}{p}}\left(\frac{1}{2}\right)^{\frac{1}{q}}\left(\left|f^{\prime \prime}(a)\right|^{q}+\left|f^{\prime \prime}(b)\right|^{q}\right)^{\frac{1}{q}} \\
& \leq \frac{\eta^{2}(b-a)}{4}\left(\frac{1}{2}\right)^{\frac{1}{q}}\left(\left|f^{\prime \prime}(a)\right|^{q}+\left|f^{\prime \prime}(b)\right|^{q}\right)^{\frac{1}{q}}
\end{aligned}
$$

where we also use the inequality (3.8) for $p>1$ and $\frac{1}{p}+\frac{1}{q}=1$.

It is noted that the result of the second inequality (3.10) is the same as the one presented by Barani, Ghazanfari, and Dragomir in [37], Theorem 4.3. Clearly, the result of the first inequality (3.10) is better than the inequality established by Barani et al. in [37], Theorem 4.3.

A different approach leads to the following results.

Theorem 3.4 Suppose that all the assumptions of Theorem 3.2 are satisfied. Then the following inequality for the Riemann-Louville fractional integral with $0<\alpha \leq 1$ holds:

$$
\begin{aligned}
& \left|R_{f}(\alpha ; \eta, m, a, b)\right| \\
& \quad \leq \frac{\eta^{2}(b, a, m)}{2(\alpha+1)}\left(\frac{\alpha}{\alpha+2}\right)^{1-\frac{1}{q}}\left[m\left(\frac{2 \alpha^{2}+\alpha-2}{(\alpha+2)(2 \alpha+2)}+\beta(\alpha+1, \alpha+2)\right)\left|f^{\prime \prime}(a)\right|^{q}\right.
\end{aligned}
$$




$$
\left.+\left(\frac{1}{2 \alpha+2}-\beta(\alpha+1, \alpha+2)\right)\left|f^{\prime \prime}(b)\right|^{q}\right]^{\frac{1}{q}}
$$

Proof Since $m a+t \eta(b, a, m) \in A$ for every $t \in[0,1]$, by utilizing the properties of modulus on Lemma 3.1 and using Hölder's integral inequality for $q>1$, we can obtain

$$
\begin{aligned}
& \left|R_{f}(\alpha ; \eta, m, a, b)\right| \\
& \leq \frac{\eta^{2}(b, a, m)}{2} \int_{0}^{1}\left|\frac{1-t^{\alpha+1}-(1-t)^{\alpha+1}}{\alpha+1}\right| f^{\prime \prime}(m a+t \eta(b, a, m)) \mid \mathrm{d} t \\
& \leq \frac{\eta^{2}(b, a, m)}{2(\alpha+1)}\left[\int_{0}^{1}\left(1-t^{\alpha+1}-(1-t)^{\alpha+1}\right) \mathrm{d} t\right]^{1-\frac{1}{q}} \\
& \quad \times\left[\int_{0}^{1}\left(1-t^{\alpha+1}-(1-t)^{\alpha+1}\right)\left|f^{\prime \prime}(m a+t \eta(b, a, m))\right|^{q} \mathrm{~d} t\right]^{\frac{1}{q}} \\
& =\frac{\eta^{2}(b, a, m)}{2(\alpha+1)}\left(\frac{\alpha}{\alpha+2}\right)^{1-\frac{1}{q}}\left[\int_{0}^{1}\left(1-t^{\alpha+1}-(1-t)^{\alpha+1}\right)\left|f^{\prime \prime}(m a+t \eta(b, a, m))\right|^{q} \mathrm{~d} t\right]^{\frac{1}{q}} .
\end{aligned}
$$

Using the generalized $(\alpha, m)$-preinvexity of $\left|f^{\prime \prime}\right|^{q}$ on $A$, we have

$$
\begin{aligned}
& \int_{0}^{1}\left(1-t^{\alpha+1}-(1-t)^{\alpha+1}\right)\left|f^{\prime \prime}(m a+t \eta(b, a, m))\right|^{q} \mathrm{~d} t \\
& \leq \int_{0}^{1}\left(1-t^{\alpha+1}-(1-t)^{\alpha+1}\right)\left(m\left(1-t^{\alpha}\right)\left|f^{\prime \prime}(a)\right|^{q}+t^{\alpha}\left|f^{\prime \prime}(b)\right|^{q}\right) \mathrm{d} t \\
& =m\left(\frac{2 \alpha^{2}+\alpha-2}{(\alpha+2)(2 \alpha+2)}+\beta(\alpha+1, \alpha+2)\right)\left|f^{\prime \prime}(a)\right|^{q} \\
& \quad+\left(\frac{1}{2 \alpha+2}-\beta(\alpha+1, \alpha+2)\right)\left|f^{\prime \prime}(b)\right|^{q} .
\end{aligned}
$$

Thus, we get the desired inequality (3.11).

Simple calculation yields the following results.

Corollary 3.6 With the same assumptions given in Theorem 3.4, if $\eta(b, a, m)=b-m a$, we obtain

$$
\begin{aligned}
& \left|\frac{f(m a)+f(b)}{2}-\frac{\Gamma(\alpha+1)}{2(b-m a)^{\alpha}}\left[J_{m a^{+}}^{\alpha} f(b)+J_{b^{-}}^{\alpha} f(m a)\right]\right| \\
& \leq \frac{(b-m a)^{2}}{2(\alpha+1)}\left(\frac{\alpha}{\alpha+2}\right)^{1-\frac{1}{q}}\left[m\left(\frac{2 \alpha^{2}+\alpha-2}{(\alpha+2)(2 \alpha+2)}+\beta(\alpha+1, \alpha+2)\right)\left|f^{\prime \prime}(a)\right|^{q}\right. \\
& \left.\quad+\left(\frac{1}{2 \alpha+2}-\beta(\alpha+1, \alpha+2)\right)\left|f^{\prime \prime}(b)\right|^{q}\right]^{\frac{1}{q}},
\end{aligned}
$$

specially for $\alpha=m=1$ and $\left|f^{\prime \prime}\right| \leq K$ on $[a, b]$, we get

$$
\left|\frac{f(a)+f(b)}{2}-\frac{1}{b-a} \int_{a}^{b} f(x) \mathrm{d} x\right| \leq \frac{(b-a)^{2}}{12} K .
$$

It is worthwhile to note that the inequality in (3.12) is better than the inequality in (3.7). 
Corollary 3.7 In Theorem 3.4 , if the mapping $\eta(b, a, m)$ with $m=1$ degenerates into $\eta(b, a)$ and we choose $\alpha=1$, then (3.11) becomes

$$
\begin{gathered}
\left|\frac{f(a)+f(a+\eta(b, a))}{2}-\frac{1}{\eta(b, a)} \int_{a}^{a+\eta(b, a)} f(x) \mathrm{d} x\right| \\
\leq \frac{\eta^{2}(b, a)}{12}\left(\frac{1}{2}\right)^{\frac{1}{q}}\left[\left|f^{\prime \prime}(a)\right|^{q}+\left|f^{\prime \prime}(b)\right|^{q}\right]^{\frac{1}{q}},
\end{gathered}
$$

which is the inequality established by Barani et al. in [37], Theorem 4.3.

Finally we shall prove the following result.

Theorem 3.5 Suppose that all the assumptions of Theorem 3.3 are satisfied. Then the following inequality for the Riemann-Louville fractional integral with $0<\alpha \leq 1$ holds:

$$
\begin{aligned}
& \left|R_{f}(\alpha ; \eta, m, a, b)\right| \\
& \leq \frac{\eta^{2}(b, a, m)}{2(\alpha+1)}\left[\frac{(q-p) \alpha-p+1}{(q-p) \alpha+2 q-p-1}\right]^{\frac{q-1}{q}} \\
& \quad \times\left\{m\left[\frac{\alpha p+\alpha+1}{(\alpha+1)(p+1)}-\frac{2}{p(\alpha+1)+1}+\beta(\alpha+1, p(\alpha+1)+1)\right]\left|f^{\prime \prime}(a)\right|^{q}\right. \\
& \left.\quad+\left[\frac{p}{(\alpha+1)(p+1)}-\beta(\alpha+1, p(\alpha+1)+1)\right]\left|f^{\prime \prime}(b)\right|^{q}\right\}^{\frac{1}{q}} .
\end{aligned}
$$

Proof Since $m a+t \eta(b, a, m) \in A$ for every $t \in[0,1]$, by using the properties of modulus on Lemma 3.1 and Hölder's integral inequality for $q>1$, we can obtain

$$
\begin{aligned}
& \left|R_{f}(\alpha ; \eta, m, a, b)\right| \\
& \leq \frac{\eta^{2}(b, a, m)}{2} \int_{0}^{1}\left|\frac{1-t^{\alpha+1}-(1-t)^{\alpha+1}}{\alpha+1}\right| f^{\prime \prime}(m a+t \eta(b, a, m)) \mid \mathrm{d} t \\
& \leq \frac{\eta^{2}(b, a, m)}{2(\alpha+1)}\left[\int_{0}^{1}\left(1-t^{\frac{q-p}{q-1}(\alpha+1)}-(1-t)^{\frac{q-p}{q-1}(\alpha+1)}\right) \mathrm{d} t\right]^{\frac{q-1}{q}} \\
& \quad \times\left[\int_{0}^{1}\left(1-t^{\alpha+1}-(1-t)^{\alpha+1}\right)^{p}\left|f^{\prime \prime}(m a+t \eta(b, a, m))\right|^{q} \mathrm{~d} t\right]^{\frac{1}{q}} \\
& =\frac{\eta^{2}(b, a, m)}{2(\alpha+1)}\left[\frac{(q-p) \alpha-p+1}{(q-p) \alpha+2 q-p-1}\right]^{\frac{q-1}{q}} \\
& \quad \times\left[\int_{0}^{1}\left(1-t^{\alpha+1}-(1-t)^{\alpha+1}\right)^{p}\left|f^{\prime \prime}(m a+t \eta(b, a, m))\right|^{q} \mathrm{~d} t\right]^{\frac{1}{q}},
\end{aligned}
$$

where we used the inequality (3.6) and the fact that

$$
\int_{0}^{1}\left(1-t^{\frac{q-p}{q-1}(\alpha+1)}-(1-t)^{\frac{q-p}{q-1}(\alpha+1)}\right) \mathrm{d} t=\frac{(q-p) \alpha-p+1}{(q-p) \alpha+2 q-p-1} .
$$


Utilizing the inequality (3.6) again and the generalized $(\alpha, m)$-preinvexity of $\left|f^{\prime \prime}\right|^{q}$ on $A$, we have

$$
\begin{aligned}
& \int_{0}^{1}\left(1-t^{\alpha+1}-(1-t)^{\alpha+1}\right)^{p}\left|f^{\prime \prime}(m a+t \eta(b, a, m))\right|^{q} \mathrm{~d} t \\
& \leq \int_{0}^{1}\left(1-t^{(\alpha+1) p}-(1-t)^{(\alpha+1) p}\right)\left(m\left(1-t^{\alpha}\right)\left|f^{\prime \prime}(a)\right|^{q}+t^{\alpha}\left|f^{\prime \prime}(b)\right|^{q}\right) \mathrm{d} t \\
& =m\left[\frac{\alpha p+\alpha+1}{(\alpha+1)(p+1)}-\frac{2}{p(\alpha+1)+1}+\beta(\alpha+1, p(\alpha+1)+1)\right]\left|f^{\prime \prime}(a)\right|^{q} \\
& \quad+\left[\frac{p}{(\alpha+1)(p+1)}-\beta(\alpha+1, p(\alpha+1)+1)\right]\left|f^{\prime \prime}(b)\right|^{q} .
\end{aligned}
$$

Using (3.15) in (3.14), we get the desired inequality (3.5).

Corollary 3.8 With the same assumptions given in Theorem 3.5, if $\eta(b, a, m)=b-m a$, we obtain

$$
\begin{aligned}
& \left|\frac{f(m a)+f(b)}{2}-\frac{\Gamma(\alpha+1)}{2(b-m a)^{\alpha}}\left[J_{m a^{+}}^{\alpha} f(b)+J_{b-}^{\alpha} f(m a)\right]\right| \\
& \leq \frac{(b-m a)^{2}}{2(\alpha+1)}\left[\frac{(q-p) \alpha-p+1}{(q-p) \alpha+2 q-p-1}\right]^{\frac{q-1}{q}} \\
& \quad \times\left\{m\left[\frac{\alpha p+\alpha+1}{(\alpha+1)(p+1)}-\frac{2}{p(\alpha+1)+1}+\beta(\alpha+1, p(\alpha+1)+1)\right]\left|f^{\prime \prime}(a)\right|^{q}\right. \\
& \left.\quad+\left[\frac{p}{(\alpha+1)(p+1)}-\beta(\alpha+1, p(\alpha+1)+1)\right]\left|f^{\prime \prime}(b)\right|^{q}\right\}^{\frac{1}{q}},
\end{aligned}
$$

specially for $\alpha=m=1$ and $\left|f^{\prime \prime}\right| \leq K$ on $[a, b]$, we get

$$
\begin{aligned}
& \left|\frac{f(a)+f(b)}{2}-\frac{1}{b-a} \int_{a}^{b} f(x) \mathrm{d} x\right| \\
& \quad \leq \frac{(b-a)^{2}}{4}\left(\frac{q-2 p+1}{3 q-2 p-1}\right)^{\frac{q-1}{q}}\left(\frac{2 p-1}{2 p+1}\right)^{\frac{1}{q}} K,
\end{aligned}
$$

where $\frac{1}{p}+\frac{1}{q}=1$ with $q>1$.

Competing interests

The authors declare that they have no competing interests.

\section{Authors' contributions}

All authors contributed equally to the writing of this paper. All authors read and approved the final manuscript.

\section{Author details}

'Department of Mathematics, College of Science, China Three Gorges University, Yichang, 443002, China. ${ }^{2}$ Department of Mathematics, Government College University, Faisalabad, Pakistan.

\section{Acknowledgements}

This work was supported by the National Natural Science foundation of China under Grant No. 61374028 and No. 11601267, Hubei Province Key Laboratory of Systems Science in Metallurgical Process of China under Grant Z201402, and the Natural Science Foundation of Hubei Province, China under Grants 2013CFA131. 


\section{References}

1. Bai, SP, Qi, F, Wang, SH: Some new integral inequalities of Hermite-Hadamard type for $(\alpha, m ; P)$-convex functions on co-ordinates. J. Appl. Anal. Comput. 6(1), 171-178 (2016)

2. Du, TS, Li, YJ, Yang, ZQ: A generalization of Simpson's inequality via differentiable mapping using extended $(s, m)$-convex functions. Appl. Math. Comput. 293, 358-369 (2017)

3. Fok, H, Vong, S: Generalizations of some Hermite-Hadamard-type inequalities. Indian J. Pure Appl. Math. 46(3), 359-370 (2015)

4. Li, YJ, Du, TS: Some improvements on Hermite-Hadamard's inequalities for s-convex functions. J. Math. Study 49(1), 82-92 (2016)

5. Li, YJ, Du, TS: Some Simpson type integral inequalities for functions whose third derivatives are $(\alpha, m)$-GA-convex functions. J. Egypt. Math. Soc. 24(2), 175-180 (2016)

6. Özdemir, ME, Avci, M, Set, E: On some inequalities of Hermite-Hadamard type via m-convexity. Appl. Math. Lett. 23(9), 1065-1070 (2010)

7. Qaisar, S, Hussain, S: Some results on Hermite-Hadamard type inequality through convexity. Turk. J. Anal. Number Theory 2(2), 53-59 (2014)

8. Sarikaya, MZ, Kiris, ME: Some new inequalities of Hermite-Hadamard type for s-convex functions. Miskolc Math. Notes 16(1), 491-501 (2015)

9. Set, E, Sardari, M, Özdemir, ME, Rooin, J: On generalizations of the Hadamard inequality for $(\alpha, m)$-convex functions. Kyungpook Math. J. 52(3), 307-317 (2012)

10. Antczak, T: Mean value in invexity analysis. Nonlinear Anal. 60, 1473-1484 (2005)

11. Weir, T, Mond, B: Pre-invex functions in multiple objective optimization. J. Math. Anal. Appl. 136(1), $29-38$ (1998)

12. Du, TS, Liao, JG, Li, YJ: Properties and integral inequalities of Hadamard-Simpson type for the generalized (s, m)-preinvex functions. J. Nonlinear Sci. Appl. 9(5), 3112-3126 (2016)

13. Miheşan, VG: A generalization of the convexity. In: Seminar on Functional Equations, Approximation and Convexity, Cluj-Napoca, Romania (1993)

14. Noor, MA: Hermite-Hadamard integral inequalities for logpreinvex functions. J. Math. Anal. Approx. Theory 2, 126-131 (2007)

15. Barani, A, Ghazanfari, AG: Some Hermite-Hadamard type inequalities for the product of two operator preinvex functions. Banach J. Math. Anal. 9(2), 9-20 (2015)

16. Latif, MA, Dragomir, SS: On Hermite-Hadamard type integral inequalities for $n$-times differentiable log-preinvex functions. Filomat 29(7), 1651-1661 (2015)

17. Li, YJ, Du, TS: A generalization of Simpson type inequality via differentiable functions using extended $(s, m)_{\phi}$-preinvex functions. J. Comput. Anal. Appl. 22(4), 613-632 (2017)

18. Wang, Y, Zheng, MM, Qi, F: Integral inequalities of Hermite-Hadamard type for functions whose derivatives are $\alpha$-preinvex. J. Inequal. Appl. 2014, 97 (2014)

19. Latif, MA, Shoaib, M: Hermite-Hadamard type integral inequalities for differentiable $m$-preinvex and $(\alpha, m)$-preinvex functions. J. Egypt. Math. Soc. 23(2), 236-241 (2015)

20. Kilbas, AA, Srivastava, HM, Trujillo, JJ: Theory and Applications of Fractional Differential Equations. Elsevier, Amsterdam (2006)

21. Sarikaya, MZ, Set, E, Yaldiz, H, Başak, N: Hermite-Hadamard's inequalities for fractional integrals and related fractional inequalities. Math. Comput. Model. 57(9-10), 2403-2407 (2013)

22. Anastassiou, GA: Generalised fractional Hermite-Hadamard inequalities involving $m$-convexity and $(s, m)$-convexity. Facta Univ., Ser. Math. Inform. 28, 107-126 (2013)

23. Chen, FX: Extensions of the Hermite-Hadamard inequality for harmonically convex functions via fractional integrals. Appl. Math. Comput. 268, 121-128 (2015)

24. Dahmani, Z, Tabharit, L, Taf, S: New generalisations of gruss inequality using Riemann-Liouville fractional integrals. Bull. Math. Anal. Appl. 2(3), 93-99 (2010)

25. Dragomir, SS, Bhatti, MI, Iqbal, M, Muddassar, M: Some new Hermite-Hadamard's type fractional integral inequalities. J. Comput. Anal. Appl. 18(4), 655-661 (2015)

26. Hwang, SR, Tseng, KL, Hsu, KC: New inequalities for fractional integrals and their applications. Turk. J. Math. 40 471-486 (2016)

27. Iqbal, M, Bhatti, MI, Nazeer, K: Generalization of inequalities analogous to Hermite-Hadamard inequality via fractional integrals. Bull. Korean Math. Soc. 52(3), 707-716 (2015)

28. İşcan, I: New general integral inequalities for quasi-geometrically convex functions via fractional integrals. J. Inequal. Appl. 2013, 491 (2013)

29. Liao, YM, Deng, JH, Wang, JR: Riemann-Liouville fractional Hermite-Hadamard inequalities. Part II: for twice differentiable geometric-arithmetically s-convex functions. J. Inequal. Appl. 2013, 517 (2013)

30. Wang, JR, Li, XZ, Fečkan, M, Zhou, Y: Hermite-Hadamard-type inequalities for Riemann-Liouville fractional integrals via two kinds of convexity. Appl. Anal. 92(11), 2241-2253 (2013)

31. Wang, J, Deng, J, Feckan, M: Hermite-Hadamard-type inequalities for $r$-convex functions based on the use of Riemann-Liouville frantional integrals. Ukr. Math. J. 65, 193-211 (2013)

32. Wang, SH, Qi, F: Hermite-Hadamard type inequalities for s-convex functions via Riemann-Liouville fractional integrals J. Comput. Anal. Appl. 22(6), 1124-1134 (2017)

33. Hussain, S, Qaisar, S: More results on Hermite-Hadamard type inequality through $(\alpha, m)$-preinvexity. J. Appl. Anal. Comput. 6(2), 293-305 (2016)

34. Qaisar, S, lqbal, M, Muddassar, M: New Hermite-Hadamard's inequalities for preinvex functions via fractional integrals. J. Comput. Anal. Appl. 20(7), 1318-1328 (2016)

35. Li, YJ, Du, TS, Yu, B: Some new integral inequalities of Hadamard-Simpson type for extended $(s, m)$-preinvex functions Ital. J. Pure Appl. Math. 36, 583-600 (2016)

36. Yang, ZQ, Li, YJ, Du, TS: A generalization of Simpson type inequality via differentiable functions using $(s, m)$-convex functions. Ital. J. Pure Appl. Math. 35, 327-338 (2015)

37. Barani, A, Ghazanfari, AG, Dragomir, SS: Hermite-Hadamard inequality for functions whose derivatives absolute values are preinvex. J. Inequal. Appl. 2012, 247 (2012) 
38. Özdemir, ME, Avci, M, Kavurmaci, H: Hermite-Hadamard-type inequalities via $(\alpha, m)$-convexity. Comput. Math. Appl. 61, 2614-2620 (2011)

39. Mohan, SR, Neogy, SK: On invex sets and preinvex functions. J. Math. Anal. Appl. 189(3), 901-908 (1995)

40. Yang, XM, Li, D: On properties of preinvex functions. J. Math. Anal. Appl. 256(1), 229-241 (2001)

Submit your manuscript to a SpringerOpen ${ }^{\circ}$ journal and benefit from:

- Convenient online submission

- Rigorous peer review

- Immediate publication on acceptance

- Open access: articles freely available online

- High visibility within the field

- Retaining the copyright to your article 